\title{
Defectos del tubo neural. Experiencia en el Instituto Nacional Materno Perinatal 2006-2010
}

\author{
Hernán Sanabria, Carolina Tarqui, Juan Arias \\ Departamento de Medicina Preventiva y Salud Pública, Facultad de Medicina, UNMSM
}

\begin{abstract}
Objetivos: Determinar la tendencia de la tasa de incidencia hospitalaria de defectos del tubo neural (DTN) y sus tipos, durante el periodo de posfortificación de la harina de trigo (2006-2010), en el INMP de Lima.

Diseño: Estudio observacional, descriptivo, retrospectivo y transversal.

Institución: Departamento de Medicina Preventiva y Salud Pública, Facultad de Medicina, UNMSM.

Participantes: Recién nacidos y sus correspondientes madres.

Intervenciones: Se revisó 88236 historias clínicas de recién nacidos (RN) y sus correspondientes madres, durante los años 2006 a 2010.

Principales medidas de resultados: Incidencia de defectos del tubo neural, después de la fortificación de la harina de trigo con ácido fólico.

Resultados: Hubo 77 RN con diversos tipos de DTN. Los DTN fluctuaron entre 2 y 9 casos por año, correspondiendo a una tasa hospitalaria global de 8,73 (IC: 6,$89 ; 10,91)$ RN. En 2006 , la incidencia fue la más alta del quinquenio $(15,63 \mathrm{x}$ 10 000; IC: 10,21; 22,89), mientras que en el 2010 la tasa fue 7,61 (IC: 4,05; 13,01). La incidencia de espina bífida fue $6,69 \times 10000$ (IC: 5,$09 ; 8,62)$, seguida de anencefalia con $1,93 \times 10000(1,12 ; 3,08)$ y encefalocele $1 \times 10000(0,10$; $0,63)$.

Conclusiones: La tendencia de la incidencia de DTN en el período 2006-2010, después de la fortificación de la harina con ácido fólico, fue descendente en el INMP de Lima.

Palabras clave: Tubo neural, malformación, ácido fólico, fortificación, incidencia.
\end{abstract}

\section{Sensibilidad insulínica en obesos y diabéticos de tipo 2 sometidos a hipoxia hipobárica intermitente}

Oscar Castillo, Elizabeth Gonzales, Lida Tello, Christopher Cárdenas, Ángela Roncal, Edgar Florentín

Instituto Nacional de Biologia Andina, UNMSM, y Hospital Nacional Arzobispo Loayza

Objetivos: Determinar la sensibilidad insulínica en obesos y diabéticos de tipo 2, bajo hipoxia hipobárica intermitente.

Diseño: Estudio comparativo clínico.

Institución: Instituto Nacional de Biología Andina, UNMSM, y Hospital Nacional Arzobispo Loayza.

Participantes: Obesos, diabéticos de tipo 2 y sanos.

Intervenciones: Se estudió 25 sujetos, sanos (8), obesos (5) y diabéticos de tipo 2 (12), sometidos a hipoxia hipobárica intermitente a 3200 m.s.n.m. en cámara hipobárica, una hora semanal por 4 semanas. Se obtuvo muestras de sangre al inicio y al finalizar la última semana. Se determinó insulina (IRMA), glicemia, colesterol, triglicéridos, HDLc, LDLC, y hemoglobina glicosilada. Se obtuvo la sensibilidad insulínica usando el método HOMA-IR y QUICKI. El análisis estadístico utilizó la prueba t Student, Tukey y el programa SPSS 15.

Principales medidas de resultados: Sensibilidad insulínica.

Resultados: Después de la cuarta semana de exposición, la sensibilidad insulínica se incrementó en los sanos y obesos. HOMA-IR: $3,17 \pm 0,49$ vs. $1,64 \pm 0,28$ ( $p=, 007)$ y QUICKI: $0,55 \pm, 025$ vs. $0,66 \pm, 028(p=, 008)$ en sujetos sanos. QUICKI: $0,49 \pm, 038$ vs. $0,59 \pm, 015(p=, 035)$ en sujetos obesos.

Conclusiones: La hipoxia intermitente en cámara hipobárica mejoró la sensibilidad insulínica en sujetos sanos y obesos.

Palabras clave: Sensibilidad insulínica, hipoxia hipobárica. 